\title{
Space weathering of Vesta and V-type asteroids: new irradiation experiments on HED meteorites ${ }^{\star}$
}

\author{
D. Fulvio ${ }^{1,2}$, R. Brunetto ${ }^{3}$, P. Vernazza ${ }^{4}$, and G. Strazzulla ${ }^{2}$ \\ 1 Laboratory for Atomic and Surface Physics, University of Virginia, Thornton Hall B-113, Charlottesville, VA 22904, USA \\ e-mail: df6vz@virginia.edu; dfu@oact.inaf.it \\ 2 INAF - Osservatorio Astrofisico di Catania, via S. Sofia 78, 95123 Catania, Italy \\ 3 Institut d'Astrophysique Spatiale, CNRS, UMR-8617, Université Paris-Sud, Bât. 121, 91405 Orsay Cedex, France \\ ${ }^{4}$ European Southern Observatory, K. Schwarzschild-Str. 2, 85748 Garching, Germany
}

Received 18 November 2011 / Accepted 27 December 2011

\begin{abstract}
Context. In the past few decades, several tens of main belt asteroids have been found to exhibit basaltic surface composition, similar to the one of Vesta and basaltic Howardite, Eucrite, and Diogenite achondrite meteorites (HEDs). Most of these objects (V-types) belong to the Vesta dynamical family. Several questions on the relationship between Vesta, V-types, and HEDs are still unresolved. In particular, Vesta is spectroscopically bluer than most V-types.

Aims. To date, it has not yet been understood whether these spectral differences are due to space weathering, similar to what has been observed for OC meteorites and S-type asteroids. To test this hypothesis, ion irradiation experiments were performed on different samples of eucrites to simulate the effects of space weathering on Vesta and V-types by solar wind ions.

Methods. Eucrite meteorites were analyzed before and after different stages of ion irradiation by VIS-NIR reflectance spectroscopy $(0.4-2.5 \mu \mathrm{m})$. We used different ions $\left(\mathrm{Ar}^{+}, \mathrm{C}^{+}\right)$with different energies (from 60 to $200 \mathrm{keV}$ ) to weather the samples.

Results. Ion irradiation was observed to alter the spectral properties of eucrite meteorites, inducing progressive reddening and darkening of the irradiated samples. Different eucrite samples (Bereba and Dar Al Gani 684) show different reddening behaviors. Because they are patchy, reddening also varied between different parts of the same meteorite. Moreover, for both meteorites, irradiation effects are much faster ( $\sim 100$ times) for $\mathrm{C}^{+}$than for $\mathrm{Ar}^{+}$ions.

Conclusions. Comparing the laboratory spectra of eucrites before and after different stages of ion irradiation with those of V-types, it turns out that the slope spread shown by V-type asteroids can be explained by space weathering. These results provide new clues to the connection between Vesta, V-types, and HEDs, and are also useful in the context of the NASA Dawn mission to Vesta.
\end{abstract}

Key words. minor planets, asteroids: individual: Vesta - techniques: spectroscopic - methods: laboratory - infrared: general

\section{Introduction}

In the study of asteroids, the term "space weathering" is used to indicate all processes able to alter the surface optical properties of bodies protected neither by atmospheres nor by magnetic fields (Hapke 2001; Chapman 2004). For most classes of asteroids and meteorites, space weathering remains poorly understood, also because of the scarcity of dedicated experiments (Lazzarin et al. 2006; Brunetto 2009; Gaffey 2010). Among these different taxonomic classes, the V-type asteroids (Binzel \& Xu 1993; DeMeo et al. 2009) are an interesting test case for space weathering studies. Many V-types belong to Vesta's dynamical family ("Vestoids") and are believed to derive from the collisional event responsible for the large crater $(460 \mathrm{~km}$ wide) near the south pole of this asteroid (Thomas et al. 1997; Nesvorný et al. 2008). The reflectance spectra of Vesta also show spectral features (and brightness) common to those of the howardite, eucrite, and diogenite achondrite meteorites (also called "HEDs"; Marzari et al. 1996; Burbine et al. 2001; Drake 2001; Pieters et al. 2006). This unique agreement is considered to be the main proof of the relationship between Vesta and these meteorites, although Vesta itself is not believed to be their

$\star$ Table 1 is available in electronic form at http://www . aanda.org direct source. The more realistic scenario considers a link between Vesta and HEDs, via basaltic fragments that reach the Earth through complex dynamical processes (see Binzel et al. 2004, and references therein). The similarity between Vesta and the HEDs' reflectance spectra also suggests that Vesta's surface is free of heavy space weathering (McCord et al. 1970; Gaffey 1983; Keil 2002; Chapman 2004; Vernazza et al. 2006).

A limited number of experiments have been conducted on HEDs to simulate space weathering on Vesta and V-type analogs. Hiroi \& Pieters (1998, and references therein) discussed $\mu$ s pulsed laser irradiation of the diogenite Johnstown: irradiation effects included darkening and reddening of the visible-NIR spectra, and weakening of the typical 1 and $2 \mu \mathrm{m}$ bands of pyroxenes (major component of the HEDs). Vernazza et al. (2006) have measured a pronounced spectral reddening and darkening by irradiating the eucrite meteorite Bereba with $400 \mathrm{keV} \mathrm{Ar}^{++}$, and consequently speculate that some mechanism (for instance, protection by a magnetic field) should be responsible for efficiently counterbalancing the reddening of Vesta's surface induced by space weathering. In fact, Vesta (as well as the HEDs) is spectroscopically bluer than the majority of V-types (Hiroi \& Pieters 1998; Marchi et al. 2010; Moskovitz et al. 2010), similar to what has been previously observed in the case of OCs/S-type asteroids (Marchi et al. 2005); however, in 

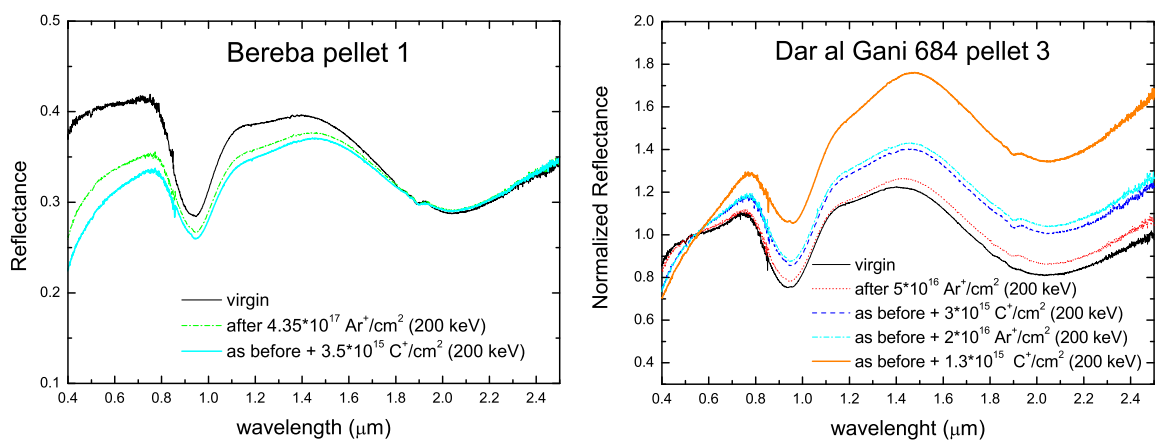

Fig. 1. VIS-NIR reflectance spectra of Bereba pellet 1 and Dar Al Gani 684 pellet 3 (normalized reflectance), before and after multiple irradiations.

a recent work, in contrast to the behavior of S-types, the visible slope of V-types was found to decrease with increasing exposure to the solar wind (Marchi et al. 2010). This slope-exposure anti-correlation seems to contradict the few laboratory results available and remains to be explained.

In this paper, we provide new laboratory data on $\mathrm{Ar}^{+}$and $\mathrm{C}^{+}$ ion irradiation on HEDs as a simulation of space weathering of V-type asteroids.

\section{Experiments}

Experiments were performed at the Laboratorio di Astrofisica Sperimentale (LASp) at INAF - Osservatorio Astrofisico di Catania (Italy). The experimental setup was composed of a stainless steel high-vacuum chamber operating at a base pressure $P<$ $10^{-7}$ mbar interfaced to an ion implanter (Danfysik 1080-200) from which ions with energy from $30 \mathrm{keV}$ up to $200 \mathrm{keV}$ (400 keV for double ionization) can be obtained. Although in the HV regime, experiments on ices and silicates performed before and after the HED experiments discussed in this paper did not ever show contaminations in our system. The experiments were performed at room temperature. In this work we collected "ex-situ" hemispherical reflectance spectra in the spectral range 0.4-2.5 $\mu \mathrm{m}$ (resolution $2 \mathrm{~nm}$ ) of unirradiated and irradiated samples using a VIS-NIR spectrometer (Perkin Elmer - Lambda 19), equipped with an integrating sphere in $\mathrm{BaSO}_{4}$. Meteorite spectra were referred to a blank spectrum of a standard $\mathrm{BaSO}_{4}$. The error in the reflectance spectra is about $5 \%$. Further details of the experimental apparatus can be found in Brunetto \& Strazzulla (2005) and Fulvio et al. (2010).

Various pellets of the eucrite meteorites Bereba and Dar Al Gani 684 (hereafter referred to as DaG) were obtained, simulating asteroidal regolith, by pressing ( $\backsim 3$ tons for $\backsim 10 \mathrm{~s}$ ) the original meteorite powder (average grain size much larger than $10 \mu \mathrm{m})$. It is widely accepted that eucrite meteorites characterize Vesta's surface well (Marzari et al. 1996; Burbine et al. 2001; Drake 2001). Spectra of Bereba and DaG show two broad bands centered around 1 and $2 \mu \mathrm{m}$ owing to pyroxene, along with a small shoulder around $1.3 \mu \mathrm{m}$ due to the presence of plagioclase.

In Sect. 3, we present the results of ion irradiation experiments on various Bereba and $\mathrm{DaG}$ pellets performed using $\mathrm{Ar}^{+}$ and $\mathrm{C}^{+}$projectiles, in the energy range $60-200 \mathrm{keV}$ (see Table 1 for a detailed list of the irradiated pellets, along with the ion, energy and fluence (ions $/ \mathrm{cm}^{2}$ ) used). The thickness of the samples is greater than the penetration depth of the used ions (on the order of $1 \mu \mathrm{m}$ ) that then remain implanted into the target. Implanted ions damage the structure of the irradiated targets and, in addition, reactive ions such as carbon have a chance of forming new bonds containing the projectile. It has been demonstrated that $\mathrm{C}$ ion implantation in silicon and silicates form $\mathrm{CO}_{2}$ and CO (Strazzulla et al. 1996), while the presence of SiC has not been yet investigated.

From the VIS-NIR spectra normalized at $0.55 \mu \mathrm{m}$, we measured the spectral slope $\left(\mu \mathrm{m}^{-1}\right)$ of the linear continuum across the $1 \mu \mathrm{m}$ absorption band (from $\backsim 0.8 \mu \mathrm{m}$ to $\sim 1.5 \mu \mathrm{m}$ ). This band is, as usual, hereafter referred to as BI. The slope of the BI band is highly representative of space weathering alteration (see Hiroi \& Sasaki 2001; Strazzulla et al. 2005). Slopes scaled to the corresponding initial value (i.e., slope of the irradiated spectrum slope of the virgin spectrum) are shown in Fig. 2 for all of the irradiated pellets, for each irradiation step (see also Table 1). For the sake of brevity, for some samples we only discuss the results of the experiments without showing the spectra.

\section{Results}

As an example of the results of the ion irradiation experiments, the lefthand panel of Fig. 1 shows the VIS-NIR $(0.4-2.5 \mu \mathrm{m})$ reflectance spectra of Bereba pellet 1 as prepared and after two irradiation steps with different ions. The righthand panel shows the reflectance spectra of DaG pellet 3, normalized at $0.55 \mu \mathrm{m}$, before and after multiple ion irradiation steps. The typical effects of ion irradiation on iron-bearing silicates are seen for all of the considered samples: spectral darkening (i.e., decreasing of the reflectance), reddening (i.e., lower reflectance at lower wavelength), and subdued absorption bands with progressive irradiation (Hapke 2001; Brunetto \& Strazzulla 2005; Strazzulla et al. 2005; Vernazza et al. 2006; Loeffler et al. 2008). The effects of different ions on $\mathrm{DaG}$ and Bereba are made clear in Fig. 2. We can see that the spectral alterations induced by $\mathrm{C}^{+}$ions are faster and more effective than in the case of $\mathrm{Ar}^{+}$.

As an example, we see that, when irradiating Bereba pellet 1 to $4.35 \times 10^{17} \mathrm{Ar}^{+} / \mathrm{cm}^{2}(200 \mathrm{keV})$ the increment in the normalized spectral slope is equal to $0.187 \mu \mathrm{m}^{-1}$. This value increases to $0.262 \mu \mathrm{m}^{-1}$ after a further irradiation of only $3.5 \times 10^{15} \mathrm{C}^{+} / \mathrm{cm}^{2}$ (200 keV) (i.e., an ion fluence two orders of magnitude less). The same trend is observed for Bereba pellet 2, where after $5 \times 10^{16} \mathrm{Ar}^{+} / \mathrm{cm}^{2}$ (200 $\left.\mathrm{keV}\right)$ the scaled slope value is equal to $0.015 \mu \mathrm{m}^{-1}$. Continuing to irradiate Bereba 2 with $\mathrm{C}^{+}$, after a fluence of $2.3 \times 10^{16} \mathrm{C}^{+} / \mathrm{cm}^{2}$, we obtain $\Delta S(\mathrm{~d}-\mathrm{a})=0.243 \mu \mathrm{m}^{-1}$ (refer to Table 1 for more details).

Similar qualitative results are found in the case of the three DaG samples, although these are more sensitive to weathering than Bereba. These differences are shown in Fig. 2. The lefthand panel shows the trend of the scaled slope as a function of fluence for all the irradiated samples. The righthand panel shows the detail of the scaled slopes as a function of fluence each time 

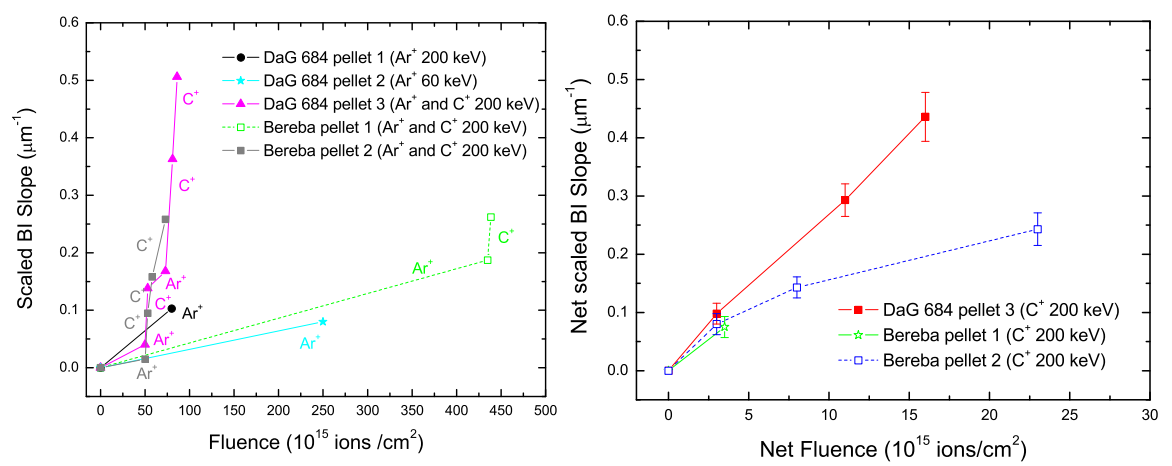

Fig. 2. Left panel: BI slope as a function of fluence for Bereba and DaG pellets after multiple irradiation steps with different ions and fluences. Each increment in the slope value is related to the ion used during irradiation (and closely labeled). Right panel: net BI slope vs. net fluence for the three eucrite pellets irradiated by $\mathrm{C}^{+}$ions. See text for further details.

that $\mathrm{C}^{+}$ion has been used as projectile. In this figure, we refer to "net" slope as the scaled slope after removing the slope variations occurring during the $\mathrm{Ar}^{+}$irradiation steps. Similarly, we refer to "net" fluence as the fluence reached considering only the fluences of the $\mathrm{C}^{+}$irradiation steps. Our experimental results can be summarized as follows.

1. The typical effects of ion irradiation on iron-bearing silicates are also seen for eucrite meteorites: spectral darkening, reddening, and subdued absorption bands with progressive irradiation.

2. The reddening is much faster ( $\sim 100$ times $)$ in the case of $\mathrm{C}^{+}$ than $\mathrm{Ar}^{+}$ions.

3. Different HEDs showed different reddening behavior. In particular, among the two eucrites considered here, DaG seems to redden faster than Bereba, when using both $\mathrm{Ar}^{+}$ or $\mathrm{C}^{+}$ions.

The last point suggests that the specificity of each eucrite, i.e., its chemical composition, presence of crystals and their structure, surface roughness and texture, is able to strongly affect the spectral changes induced by space weathering. The greater efficiency of carbon with respect to argon ions might be due to the formation of chemical bonds between the atoms in the target (mostly $\mathrm{Si}$ and $\mathrm{O}$ ) and the implanted carbon ions. In fact, it has been demonstrated that in some instances reactive ions (e.g. H, C, N, $\mathrm{O})$ form molecular bonds as is the case for $\mathrm{SiH}$ and $\mathrm{SiC}$ formed after implantation of $\mathrm{H}$ and $\mathrm{C}$, respectively, in silicon wafers. Implantation of $\mathrm{C}$ in silicates forms $\mathrm{CO}_{2}$ and $\mathrm{CO}$, while the presence of $\mathrm{SiC}$ has not been yet investigated (Strazzulla et al. 1996). In addition, implanted carbon ions could form nanoclusters that act as "colorants". The noteworthy "redness" of carbon-based materials produced by ion irradiation of frozen C-rich ices (e.g., methane, benzene, methanol) (Brunetto et al. 2006) or polymers (Kanuchova et al. 2010) is well known.

\section{Implications for V-types and Vesta}

The energy values used in the ion irradiation experiments discussed in this work are higher than that of $1 \mathrm{keV} / \mathrm{u}$ solar wind $\mathrm{Ar}$ $(36 \mathrm{keV})$ and $\mathrm{C}(12 \mathrm{keV})$ ions. We thus need to scale the results obtained in the laboratory to evaluate the timescales needed to obtain the same effects in space. For unreactive ions (Ar, in this case) it has been suggested that spectral alteration is determined by the energy deposited by incoming ions through elastic collisions with target nuclei (Brunetto \& Strazzulla 2005).

The astrophysical timescale for the solar wind ions to induce the observed spectral reddening on an asteroid surface at
2.36 AU (average heliocentric distance of asteroid Vesta) can be estimated by dividing the fluence by the solar wind $\operatorname{Ar}(36 \mathrm{keV})$ ion flux at $2.36 \mathrm{AU}$. (36 keV-Ar ion flux at $1 \mathrm{AU}$ is about $4 \times 10^{2}$ ions cm $\mathrm{cm}^{-2} \mathrm{~s}^{-1}$, Jull et al. 1980; this value is then scaled at 2.36 AU with a variation as the inverse of the squared heliocentric distance.) Then the timescale is corrected to take into account that the elastic collision damage of $200 \mathrm{keV}$ Ar ions is about four times stronger than $36 \mathrm{keV}$ Ar ions, as well as the ratio between the geometrical cross-section and the whole asteroidal surface (a factor of 4) assuming a uniformly irradiated spherical body (Vernazza et al. 2006). Since Ar is a minor component of the solar wind, we should consider all other solar wind ions $(\mathrm{H}, \mathrm{He}, \mathrm{O}, \mathrm{N}$, etc.) that may contribute to altering the surface. Again using the elastic collision damage, we estimate a correction factor of about 1000 (Vernazza et al. 2006). With this in mind, we evaluate that a laboratory fluence of $10^{15}$ $200 \mathrm{keV}$ Ar ions $\mathrm{cm}^{-2}$ corresponds to about $7 \times 10^{3} \mathrm{yrs}$. The flux of solar wind $\mathrm{C}$ ions at $2.36 \mathrm{AU}$ is about $8.6 \times 10^{3}$ ions $\mathrm{cm}^{-2} \mathrm{~s}^{-1}$ (Jull et al. 1980). A laboratory fluence of $10^{15} \mathrm{C}_{\text {ions }} \mathrm{cm}^{-2}$ corresponds to about $1.4 \times 10^{4}$ yrs at Vesta. Because the reddening induced by carbon ions is significantly higher than that of Ar, we believe that carbon ions (and possibly other ions such as sulfur) are the main agents in reddening the basaltic surfaces directly exposed to the solar wind. To fully understand whether the proposed mechanisms underlie the observed spectral alterations, more experiments should be done employing an extended set of ions ( $\mathrm{He}, \mathrm{O}, \mathrm{N}$, etc.) and energies.

To better understand the connection between Vesta, V-type asteroids, and HED meteorites, we now compare (Fig. 3) the laboratory spectra of $\mathrm{DaG}$ and Bereba before and after different stages of $\mathrm{Ar}^{+}$and $\mathrm{C}^{+}$irradiation with those of Vesta and some V-type asteroids (covering the whole range of spectral slopes observed for this class of asteroids; spectra from SMASS and SMASSIR surveys: http://smass.mit.edu). In the lefthand panel, we consider only the $\mathrm{Ar}^{+}$irradiation stages. In the righthand one, we also consider the $\mathrm{C}^{+}$ones. It is clear that, at the fluences reached in our experiments, the $\mathrm{Ar}^{+}$irradiation experiments (left panel) are not able to cover the whole range of BI slopes observed for the V-types asteroids. Even when starting with the reddest virgin spectra ( $\mathrm{DaG}$ pellet 2 and $\mathrm{DaG}$ pellet 3), we are able to cover no more than half of the V-types' BI slopes after the maximum irradiation fluences. The maximum reddening is observed for $\mathrm{DaG}$ pellet 3 after a space weathering timescale of $\sim 5 \times 10^{5}$ years (corresponding to a laboratory fluence of $7 \times 10^{16} 200 \mathrm{keV} \mathrm{Ar}$ ions $\mathrm{cm}^{-2}$ ). In the case of Bereba pellet 1 , the maximum reddening reached after $\sim 3 \times 10^{6}$ years is even less. This is because of the lower starting slope and slower 

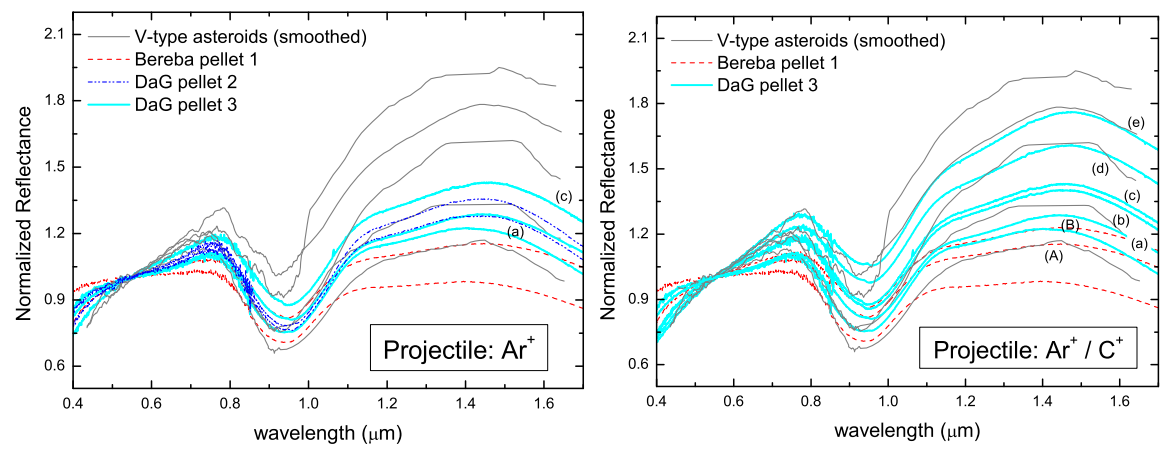

Fig. 3. Comparison between VIS-NIR spectra of V-type asteroids with spectra of DaG and Bereba at different stages of irradiation. For each sample the lowest spectrum is the virgin one. Labels refer to the irradiation stages reported in Table 1. Left panel: Ar ${ }^{+}$irradiated samples. Right panel: $\mathrm{Ar}^{+}$and $\mathrm{C}^{+}$irradiated samples. See text for details.

reddening rate of Bereba with respect to $\mathrm{DaG}$. The same trend is seen for Bereba pellet 2 (not shown for the sake of clarity).

When taking the $\mathrm{C}^{+}$irradiated samples into account, the results are different. In the righthand panel of Fig. 3, we can see that starting with $\mathrm{DaG}$ meteorite allows us to cover the whole range of V-types' BI slopes within a space weathering timescale $\sim 2.5 \times 10^{5}$ years (corresponding to a laboratory fluence of $1.6 \times 10^{16} 200 \mathrm{keV} \mathrm{C}$ ions $\mathrm{cm}^{-2}$ ). Although there are experimental limitations that do not allow us to easily simulate space weathering timescales longer than $10^{5}-10^{7}$ years, we showed that these timescales are long enough for V-type asteroids experiencing strong reddening. In other words, space weathering may be able to alter their surface and reproduce the whole range of observed V-types' spectral slopes, different slopes mirroring different degrees of contribution of intrinsic (chemical composition, surface roughness, texture, etc.) and extrinsic factors (presence of "colorants", ion irradiation timescale, etc.).

The results presented in this paper are relevant for better understanding the link between Vesta, V-types, and HEDs. They are also useful in the framework of the observed presence of Vtypes asteroids that do not belong to the Vesta dynamical family (DeSanctis et al. 2011; Fulvio et al. in prep.). The analysis of the spectra of basaltic asteroids that are not directly connected to Vesta can provide insight into the question of the relevance of the space weathering as a function of the distance from the Sun. They will also be useful in the context of the NASA Dawn mission, which has been in orbit around Vesta since July 2011 (Russell et al. 2007; Pieters et al. 2011). Preliminary data from Dawn seem to confirm that a variety of colors exist in different regions of Vesta, which is, however, on average, bluer than the majority of V-types. Vernazza et al. (2006) speculate that protection by a magnetic field could avoid direct exposure of most of Vesta's surface to solar wind weathering. This seems to be supported by recent studies of paleomagnetism of the Millbillillie Eucrite, which produced evidence of remanent magnetism recorded on its parent body Vesta (Fu \& Weiss 2011). Other data from the Hubble Space Telescope show a lack of correlations between albedo and the spectral slope that points towards the absence of globalized space weathering ( $\mathrm{Li}$ et al. 2010). However, the same authors find evidence of space weathering in some regions, including the bright rim of the south pole crater.

To help the community interpret the Dawn data, we provide free access to the spectra of irradiated HEDs at the LASp website: http://web.ct.astro.it/weblab/dbindex.html.
Acknowledgements. We thank the Muséum National d'Histoire Naturelle for providing us with the meteoritic samples. We also thank R. A. Baragiola, G. A. Baratta, and M. E. Palumbo for their useful suggestions and discussions, F Spinella for his help to perform the experiments, and Emma Mitchell for editing the manuscript.

\section{References}

Binzel, R. P., \& Xu, S. 1993, Science, 260, 186

Binzel, R. P., Rivkin, A. S., Stuart, J. S., et al. 2004, Icarus, 170, 259

Brunetto, R. 2009, Earth Moon and Planets, 105, 249

Brunetto, R., \& Strazzulla, G. 2005, Icarus, 179, 265

Brunetto, R., Barucci, M. A., Dotto, E., \& Strazzulla, G. 2006, ApJ, 644, 646

Burbine, T. H., Buchanan, P. C., Binzel, R. P., et al. 2001, Meteor. Planet. Sci., 36,761

Chapman, C. R. 2004, Ann. Rev. Earth Planet. Sci., 32, 539

DeMeo, F. E., Binzel, R. P., Slivan, S. M., \& Bus, S. J. 2009, Icarus, 202, 160

De Sanctis, M. C., Ammannito, E., Migliorini, A., et al. 2011, MNRAS, 412, 2318

Drake, M. J. 2001, Meteor. Planet. Sci., 36, 501

Fu, R., \& Weiss, B. P. 2011, EPSC-DPS2011-1646

Fulvio, D., Guglielmino, S., Favone, T., \& Palumbo, M. E. 2010, A\&A, 511, A62 Gaffey, M. J. 1983, in Lunar and Planetary Institute Conference Abstracts, 231 Gaffey, M. J. 2010, Icarus, 209, 564

Jull, A. J. T., Wilson, G. C., Long, J. V. P., Reed, S. J. B., \& Pillinger, C. T. 1980, Nucl. Instrum. Methods, 168, 357

Hapke, B. 2001, J. Geophys. Res., 106, 10039

Hiroi, T., \& Pieters, C. M. 1998, Antarctic Meteorite Research 11, 163

Hiroi, T., \& Sasaki, S. 2001, Meteorit. Planet. Sci., 36, 1587

Kanuchova, Z., Baratta, G. A., Garozzo, M., \& Strazzulla, G. 2010, A\&A, 517, A60

Keil, K. 2002, Asteroids III, 573

Lazzarin, M., Marchi, S., Moroz, L. V., et al. 2006, ApJ, 647, L179

Li, J.-Y., McFadden, L. A., Thomas, P. C., et al. 2010, Icarus, 208, 238

Loeffler, M. J., Dukes, C. A., \& Baragiola, R. A. 2009, J. Geophys. Res., 114, E03003

Marchi, S., Brunetto, R., Magrin, S., Lazzarin, M., \& Gandolfi, D. 2005, A\&A, 443, 769

Marchi, S., De Sanctis, M. C., Lazzarin, M., \& Magrin, S. 2010, ApJ, 721, L172

Marzari, F., Cellino, A., Davis, D. R., et al. 1996, A\&A, 316, 248

McCord, T. B., Adams, J. B., \& Johnson, T. V. 1970, Science, 168, 1445

Moskovitz, N. A., Willman, M., Burbine, T. H., Binzel, R. P., \& Bus, S. J. 2010, Icarus, 208, 773

Nesvorný, D., Roig, F., Gladman, B., et al. 2008, Icarus, 193, 85

Pieters, C. M., Binzel, R. P., Bogard, D., et al. 2006, Asteroids, Comets, Meteors, 273, Proc. 229th Symp. IAU, Rio de Janeiro-Brasil, 2005

Pieters, C. M., McFadden, L. A., Prettyman, T., et al. 2011, Space Sci. Rev., on-line version

Russell, C. T., Capaccioni, F., Coradini, A., et al. 2007, Earth Moon Planets, 101, 65

Strazzulla, G., Brucato, J. R., Palumbo, M. E., \& Satorre, M. A. 1996, Nucl. Instr. Meth. B, 116, 289

Strazzulla, G., Dotto, E., Binzel, R., et al. 2005, Icarus, 174, 31

Thomas, P. C., Binzel, R. P., Gaffey, M. J., et al. 1997, Science, 277, 1492

Vernazza, P., Brunetto, R., Strazzulla, G., et al. 2006, A\&A, 451, L43 
D. Fulvio et al.: New irradiation experiments on HED meteorites

Table 1. Bereba and Dar Al Gani 684 (here referred to as DaG) pellets along with used ions, energy, total fluence, BI slopes scaled to the initial value (i.e., slope of the irradiated spectrum - slope of the virgin one), and BI slope error.

\begin{tabular}{|c|c|c|c|c|c|}
\hline Pellet & Ion & $\begin{array}{c}\text { Energy } \\
(\mathrm{keV})\end{array}$ & $\begin{array}{l}\text { Tot. fluence } \\
\text { (ions } / \mathrm{cm}^{2} \text { ) }\end{array}$ & $\begin{array}{l}\text { Scaled BI Slope } \\
\qquad\left(\mu \mathrm{m}^{-1}\right)\end{array}$ & $\begin{array}{l}\text { Scaled BI Slope error } \\
\left(\mu \mathrm{m}^{-1}\right)\end{array}$ \\
\hline $\mathrm{DaG} 1$ & $\mathrm{Ar}^{+}$ & 200 & $8 \times 10^{16}$ & 0.103 & 0.015 \\
\hline $\mathrm{DaG} 2$ & $\mathrm{Ar}^{+}$ & 60 & $2.5 \times 10^{17}$ & 0.08 & 0.015 \\
\hline $\begin{array}{l}\text { Bereba } 1 \text { (a) } \\
\text { Bereba } 1 \text { (b) }\end{array}$ & $\begin{array}{l}\mathrm{Ar}^{+} \\
\mathrm{C}^{+} \\
\end{array}$ & $\begin{array}{l}200 \\
200\end{array}$ & $\begin{array}{c}4.35 \times 10^{17} \\
\text { a.b. }+3.5 \times 10^{15}\end{array}$ & $\begin{array}{l}0.187 \\
0.262\end{array}$ & $\begin{array}{l}0.010 \\
0.015\end{array}$ \\
\hline $\begin{array}{l}\text { Bereba } 2 \text { (a) } \\
\text { Bereba } 2 \text { (b) } \\
\text { Bereba } 2 \text { (c) } \\
\text { Bereba } 2 \text { (d) }\end{array}$ & $\begin{array}{l}\mathrm{Ar}^{+} \\
\mathrm{C}^{+} \\
\mathrm{C}^{+} \\
\mathrm{C}^{+} \\
\end{array}$ & $\begin{array}{l}200 \\
200 \\
200 \\
200 \\
\end{array}$ & $\begin{array}{c}5 \times 10^{16} \\
\text { a.b. }+3 \times 10^{15} \\
8 \times 10^{15} \\
2.3 \times 10^{16} \\
\end{array}$ & $\begin{array}{l}0.015 \\
0.095 \\
0.158 \\
0.258 \\
\end{array}$ & $\begin{array}{l}0.010 \\
0.014 \\
0.013 \\
0.018 \\
\end{array}$ \\
\hline $\begin{array}{l}\text { DaG } 3(\mathrm{a}) \\
\text { DaG } 3(\mathrm{~b}) \\
\text { DaG } 3(\mathrm{c}) \\
\text { DaG } 3(\mathrm{~d}) \\
\text { DaG } 3(\mathrm{e})\end{array}$ & $\begin{array}{c}\mathrm{Ar}^{+} \\
\mathrm{C}^{+} \\
\mathrm{Ar}^{+} \\
\mathrm{C}^{+} \\
\mathrm{C}^{+}\end{array}$ & $\begin{array}{l}200 \\
200 \\
200 \\
200 \\
200\end{array}$ & $\begin{array}{c}5 \times 10^{16} \\
\text { a.b. }+3 \times 10^{15} \\
\text { a.b. }+2 \times 10^{16} \\
\text { a.b. }+8 \times 10^{15} \\
1.3 \times 10^{16}\end{array}$ & $\begin{array}{l}0.040 \\
0.138 \\
0.168 \\
0.363 \\
0.506\end{array}$ & $\begin{array}{l}0.015 \\
0.015 \\
0.014 \\
0.019 \\
0.027\end{array}$ \\
\hline
\end{tabular}

Notes. When considering consecutive irradiation steps with the same ion, the total fluence reached with that ion is reported. When a pellet was consecutively irradiated with different ions, "a.b." (=as before) appears in the row where the irradiation with the new ion is considered. In that case, the fluence following the "a.b." label is the one with the new ion (to be added to the previous value). 